\title{
Galactic spiral generation in tidal encounters
}

\author{
Alex R. Pettitt ${ }^{1}$, Elizabeth J. Tasker ${ }^{1}$ and James W. Wadsley ${ }^{2}$ \\ ${ }^{1}$ Department of Physics, Faculty of Science, Hokkaido University, \\ Sapporo 060-0810, Japan \\ email: alex@astro1.sci.hokudai.ac.jp, tasker@astro1.sci.hokudai.ac.jp \\ ${ }^{2}$ Department of Physics and Astronomy, McMaster University, \\ Hamilton, Canada \\ email: wadsley@mcmaster.ca
}

\begin{abstract}
The existence of grand design spiral galaxies in the universe is still a standing problem. The passage of a small companion is known to be able to induce spiral structures in disc galaxies, but there remains questions over how relevant this mechanism is to the galaxies observed in the real universe. Our study aims to address two key points regarding such interactions; the limiting mass companion needed to drive tidal spiral structures, and the differences between the resulting gas and stellar morphology. We find the minimum mass of a companion to be as low as $5 \%$ of the stellar mass of the galaxy, and that the arms formed in the gas and the stars display very minor dynamical and morphological differences.
\end{abstract}

Keywords. hydrodynamics, methods: numerical, ISM: kinematics and dynamics, galaxies: structure, galaxies: spiral, galaxies: interactions

\section{Introduction}

A number of different mechanisms have been suggested to underpin galactic spiral features; density waves, swing amplified transient spirals, bar driven arms and tidal encounters. The regimes where each of these mechanisms becomes dominant is an unknown, when does an interaction between a galaxy and a companion become strong enough to drown out the structure formed in isolation? Long-lived, two-armed spirals have been difficult to generate in isolation in numerical simulations without bars or tidal encounters (e.g. Tooter \& Toomre 1972). The poster child of two-armed spirals, M51, is believed to be the result of an interaction (Dobbs et al.2010), however other unbarred two-armed spirals such as M74, are not obviously influenced by tidal interactions. In this study we perform numerous simulations of galaxies under the influence passing companions, to study the different interstellar medium (ISM) gas and stellar responses, and find the lower mass limit on the companion required to induce spiral features.

\section{Simulations}

We perform simulations using the smoothed hydrodynamics $+N$-body code GASOLINE (Wadsley et al.2004) where the galaxy is composed of a live stellar disc, bulge, dark matter halo and warm isothermal gas disc. The rotation curve of the galaxy is designed to prohibit bar formation and display a flocculent arm structure in isolation. The perturbing companion is initially $150 \mathrm{kpc}$ away from the host galaxy and is represented by a point mass particle. Numerous simulations were performed, using different mass companions and differing orbital paths.

\section{Results}

In Figure 1 we show various properties the spiral arms in our fiducial simulation in the stars (blue) and gas (red). In the left panel is the pattern speed as a function of radius, showing a clear tracing of the inner frequencies of $\Omega-\kappa / 2$, where $\Omega$ is the orbital frequency of the disc material and $\kappa$ the epicycle frequency. The spiral arms are therefore not quite material (which 

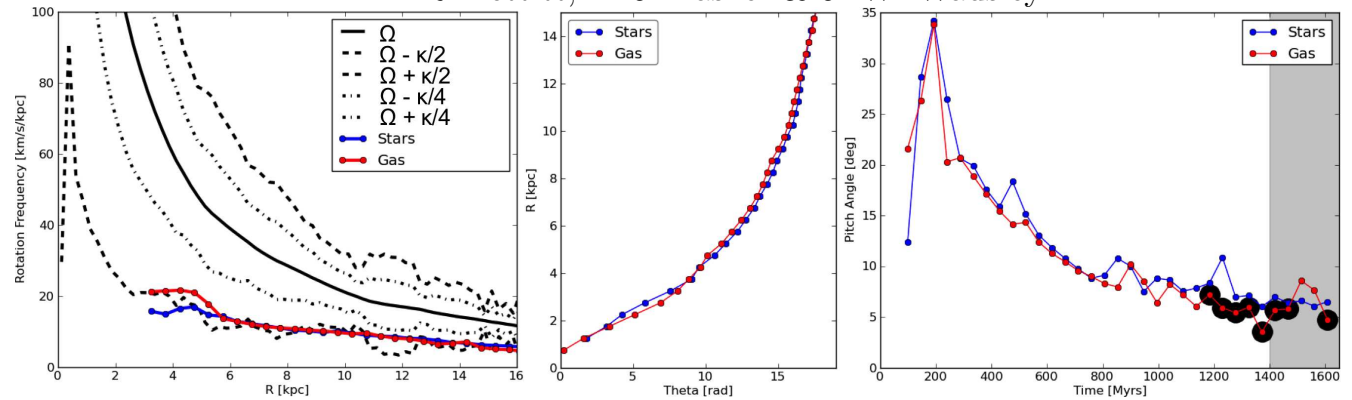

Figure 1. Various properties of our fiducial calculation, with the stellar spirals arms in blue and the gas in red. In the first panel we show the pattern speed alongside the rotation frequencies of the galaxy. The central panel shows the offset between the gas and stellar spiral arms. In the right panel is the time-evolution of the pitch angle of spiral arms, which clearly shows them winding up.

would trace $\Omega$ exactly) or steady density waves (which would have a constant pattern speed with radius). The central panel shows the offset between gas and stars in the spiral arms, showing a very weak offset in the mid-outer disc. There is also a slight migration of gas towards the galactic centre during the interaction, which is not seen in the stellar component (not pictured). The right figure shows the pitch angle of the arms displaying a strong time dependance, indicating they are winding up over the time of approximately 1 Gyr. The pitch angles span values of $5^{\circ}$ to $35^{\circ}$, a range similar to that seen in observed galaxies (e.g. Kennicutt 1981).

In our parameter sweep of different companion masses, we find the properties shown in Figure 1 are effectively universal for non-merging interactions. The behaviour of the pattern speed and pitch angle in particular are the same regardless of the mass and orbit of the companion. As the mass of the companion is reduced, so is the response of the host galaxy. We find a minimum mass of approximately $5 \%\left(\approx 1 \times 10^{9} M_{\odot}\right)$ of that of the stellar mass of the galaxy is needed to form some spiral structure. The disc response is also reduced as the orbital path becomes more retrograde with respect to galactic rotation. This minimum mass is within the range of dark matter subhalos formed in cosmological simulations and the mass of some satellites of the Milky Way (e.g. the Large Magellanic Cloud).

\section{References}

Dobbs C. L., Theis C., Pringle J. E., Bate M. R., 2010, MNRAS, 403, 625

Kennicutt R. C., 1981, AJ, 86, 1847

Toomre A., Toomre J., 1972, APJ, 178, 623

Wadsley J. W., Stadel J., Quinn T., 2004, New Astron., 9, 137 